\title{
A COMPARISON BETWEEN CONTINUOUS AND DISCRETE OPTIMAL CONTROL OF A GANTRY CRANE
}

\author{
Nazemizadeh M. ${ }^{1}$ \\ ${ }^{1}$ Department of mechanical engineering, Damavand Branch, Islamc Azad University, Damavand, Iran. \\ Email: ${ }^{1}$ mn.nazemizadeh@gmail.com
}

\begin{abstract}
This paper presents a comparison study on continuous and discrete optimal control of a gantry crane. Hence, the nonlinear dynamic model of the system is derived via Lagrange's principle, the linearized equations of the system is expressed. The motor voltage of and displacement of the trolley are presumed as input and output of the system respectively, and the state-space equations of the system are presented. Then, optimal continuous and discrete optimal feedback control law is obtained for tracking of trolley, and some tracking simulation are performed which compare the results of continuous and discrete optimal law.
\end{abstract}

Key words:continuous, discrete, optimal control, gantry crane, tracking,

\section{INTRODUCTION}

Cranes are used in various industries such as transportation and construction tasks. A crane often includes a hoisting mechanism which is suspended from a point on the support mechanism. Based on the support mechanism, cranes can be classified as: gantry (overhead) cranes, tower (rotary) crane, and boom carne. Gantry cranes are commonly composed of a cart moving in a fixed support, while a cable is suspended from a point on the cart to transport the payload. The gantry cranes have some advantages other the other models of the crane such as low cost, easy assembly and less maintenance, and have attracted a great deal of interests [1, 2]. Thus, the dynamic analysis of such a system is an important task, and treated by researchers, recently. For the modeling of the crane, two approaches are usually used including a lumped-mass or a distributed-mass modeling. For the lumped-mass modeling of the crane, the system is modeled by a massless cable as a hoisting line and a lumped mass as a payload [3-5]. On the other hand, in the distributed-mass modeling, the hoisting line is modeled as a continuous string, and the payload assumed a lumped mass as a boundary condition of the system [6, 7]. Hubbel et al. [8] used an open-loop continuous control named input-shaping to control the motion of a gantry crane. In this method, the input control profile is determined as unwanted oscillation during travel and residual pendulations are avoided [9]. Also, a hybrid input-shaping strategy and a continuous PD-type fuzzy logic control scheme are implemented in [10] to control a gantry crane system. however this method if effective, but the input-shaping method leaks from being an open loop control scheme, and is not robust to disturbances and parameter uncertainties [4]. Moustafa and Ebied [11] used a nonlinear modeling and anti-swing control method for the overhead cranes. Also, in [12] a fuzzy logic feedback controller is proposed to control an intelligent crane system.

This paper concerned with the continuous and discrete optimal control of a gantry crane. To do this, the nonlinear and linear dynamic equations of the system are derived. Then, the continuous and discrete models of the system are presented in state-space form. As the problem is the tracking of the cart position, the optimal control law in both of the continuous and discrete form is formulated, and the optimal control feedback gains are obtained. Then, some simulation results are presented to compare the continuous and discrete optimal control law.

\section{DYNAMIC MODELING}

In this section, dynamic model of the gantry carne is presented. The dynamic equation of the system is derived using Lagrange principle. Figure 1 shows a gantry crane moves in two-dimensional space. The crane is consists of a cart (trolley) transverses in horizontal direction, while a massless pendulum connects on the cart and hoists the payload.

As, the Lagrange principle is used, the lagrangian function can be stated as: 


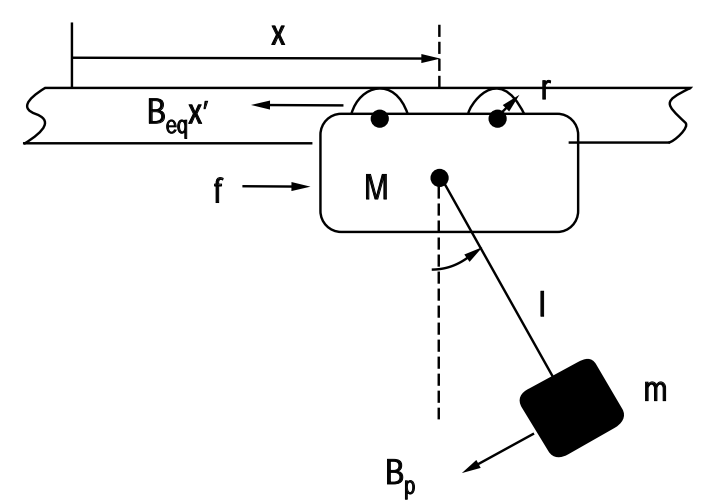

Fig. 1. The Gantry Crane

$L=\frac{1}{2} m\left[\dot{x}^{2}+\dot{P}^{2}+(\dot{\theta})^{2}+2 \dot{x} \dot{I} \sin \theta+2 \dot{x} \mid \dot{\theta} \cos \theta\right]$

$+\frac{1}{2} M \dot{x}^{2}+m g l \cos \theta$

Here in, the parameters of the overhead system are presented in Table 1:

Table 1. Parameters of the gantry crane

\begin{tabular}{|l|c|}
\hline \multicolumn{1}{|c|}{ Parameters } & Nomenclatures \\
\hline Cart position & $x$ \\
\hline Cart velocity & $\dot{x}$ \\
\hline Pendulum angular displacement & $\theta$ \\
\hline Pendulum angular velocity & $\theta$ \\
\hline Pendulum length & $l$ \\
\hline Mass of the cart system & $M$ \\
\hline Payload mass & $m$ \\
\hline Gravitational constant of earth & $g$ \\
\hline Radius of wheels of cart & $r$ \\
\hline DC motor voltage of cart & $e$ \\
\hline Force exerted to cart & $f$ \\
\hline Motor armature resistance & $R$ \\
\hline Motor torque constant & $k$ \\
\hline $\begin{array}{l}\text { Viscous damping coefficient of } \\
\text { pendulum axis }\end{array}$ & $B_{p}$ \\
\hline $\begin{array}{l}\text { Equivalent viscous damping } \\
\text { coefficient }\end{array}$ & $B_{e q}$ \\
\hline
\end{tabular}

The principle of Lagrange for dynamic systems is expressed as:

$$
\frac{d}{d t}\left(\frac{\partial L}{\partial \dot{q}_{j}}\right)-\frac{\partial L}{\partial_{q j}}=Q_{j}-Q_{j \text { lost }}
$$

Where represents the generalized coordinates, $q_{j}$ is the generalized external force, and $Q_{j}$ is defined as the generalized force related to the viscous damping of the system. The generalized coordinates are treated as $q_{1}=x, q_{2}=0$. Thus, the nonlinear equations of the system are expressed as:

$$
\begin{aligned}
& (M+m) \dot{x}+m l\left(\dot{\theta} \cos \theta-\dot{\theta}^{2} \sin \theta\right)+ \\
& +2 m i \dot{\theta} \cos \theta+m \dot{s} \sin \theta=f-B_{e q} \dot{x} \\
& I \dot{\theta}+2 \dot{l} \dot{\theta}+\dot{x} \cos \theta+g \sin \theta=-B_{p} \dot{\theta}
\end{aligned}
$$

Beside, the linear force is originated from the torque of motor of trolley [13]:

$$
\begin{aligned}
& T=r f \\
& T=\frac{k}{R} e-\frac{k^{2}}{R} \omega \\
& \dot{x}=r \omega
\end{aligned}
$$

Thus, from Eq. (3) and Eq. (4), the nonlinear model of the system is: ]:

$$
\begin{aligned}
& (M+m) \dot{x}+m / \dot{\theta} \cos \theta- \\
& -m / \dot{\theta}^{2} \sin \theta=\frac{1}{r}\left(\frac{k}{R} e-\frac{k^{2}}{R_{r}} \dot{x}\right) \\
& \dot{x} \cos \theta+l \dot{\theta}+g \sin \theta=0
\end{aligned}
$$

Also, if the state vector is defined as $X=[x \dot{x} \theta \dot{\theta}]$, and the continuous dynamic model of the system is linearized, then the continuous linear system can be expressed as:

$$
\begin{aligned}
& \dot{X}=A X+B u \\
& y=C X+D u
\end{aligned}
$$

Where the matrices of the continuous model are: 


$$
\begin{aligned}
& A=\left[\begin{array}{rrrr}
0 & 1 & 0 & 0 \\
0 & -\frac{k^{2}}{R_{r}^{2} M} & \frac{m g}{M} & 0 \\
0 & 0 & 0 & 1 \\
0 & \frac{k^{2}}{R_{r}^{2} M l} & \frac{(M+m) g}{M l} & 0
\end{array}\right] \\
& B=\left[\begin{array}{c}
0 \\
\frac{k}{R_{r} M} \\
0 \\
\frac{k}{R r M l}
\end{array}\right] C=\left[\begin{array}{llll}
1 & 0 & 0 & 0
\end{array}\right] D=0
\end{aligned}
$$

To express the discrete model of the system, one can assume the sampling time as $T$, and use the function d2C in MATLAB [14], to descretize the system model given by Eq. (6). Thus the discrete model of the system is:

$$
\begin{aligned}
X((k+1) T) & =G X(k T)+H u(k T) \\
y(k T) & =E X(k T)+F u(k T)
\end{aligned}
$$

\section{OPTIMAL CONTROL OF SYSTEM}

Consider the continuous system is given by Eq. (6). In tracking problem, It is desired that the cart tracks the desired input $r(t)$. To express the state-space equations of the system about the refrence input, it is considered that in steady state, the system equations are:

$$
\begin{aligned}
0 & =A X_{e}+B u_{e} \\
r(t) & =C X_{e}
\end{aligned}
$$

Thus, from Eqs. (6), (9), the state-space equation of the continuous system can be stated as:

$$
\begin{aligned}
& \bar{X}=A \bar{X}+B \bar{u} \\
& y=C \bar{X}
\end{aligned}
$$

Where $\bar{X}=X-X_{e}, \bar{u}=u-u_{e}, \bar{y}=y-r$.
Thus the cost function of the tracking problem of the continuous system is expressed as:

$$
\left.J=\int_{0}^{\infty} \bar{X} T Q \bar{X}+u^{T} R u\right) d t
$$

And, the optimal feedback law is $u=K \bar{X}=R^{1} B^{T} P \bar{X}$, where can be achieved from Riccati's equation [15]:

$$
A^{T} P+P A-P B R^{-1} B^{T} P+Q=0
$$

For the discrete system, if the state-space equation of the system is given by Eq. (8), then the cost function of the discrete system is:

$$
J=\frac{1}{2} \sum_{k=0}^{\infty}[X(k) Q X(k)+u(k) R u(k)]
$$

And the optimal feedback law is $u=-K X(k)=-\mid\left(R+H^{T} P H\right)^{-1} H^{T} P G X(k), \quad$ where must be satisfied the following equation [14]:

$$
-P+G^{T} P\left(I+H R^{-1} H^{T} P\right)^{-1} G+Q=0
$$

\section{SIMULATION RESULTS}

In this section, the optimal control results of the continuous and discrete systems are compared to each others. The discrete system is considered for the sampling time $T=0.1,0.5$ or $1 \mathrm{~s}$. the parameter values of the system is given asin Table 2 .

Table 2. Parameter values of the gantry crane [16]

\begin{tabular}{|l|l|}
\hline \multicolumn{1}{|c|}{ Parameter } & Parameter values \\
\hline Pendulum length & $I=0.3302 \mathrm{~m}$ \\
\hline Mass of the cart system & $M=1.073 \mathrm{~kg}$ \\
\hline Payload mass & $m=0.23 \mathrm{~kg}$ \\
\hline Gravitational constant of earth & $g=9.81 \mathrm{~m} / \mathrm{s}^{2}$ \\
\hline Radius of wheels of cart & $r=0.006 \mathrm{~m}$ \\
\hline Motor armature resistance & $R=2.6 \Omega$ \\
\hline Motor maximum voltage & $e_{\max }=12 \mathrm{~V}$ \\
\hline Motor torque constant & $k=0.00767 \mathrm{Vs} / \mathrm{rad}$ \\
\hline
\end{tabular}


As the weighting matrices are assumed as, the optimal feedback law of the system is obtained from Eq. (12) or Eq. (14).

The simulation results for the cart position, pendulum angle, and the input voltage are shown in fig 2,3 and 4 .

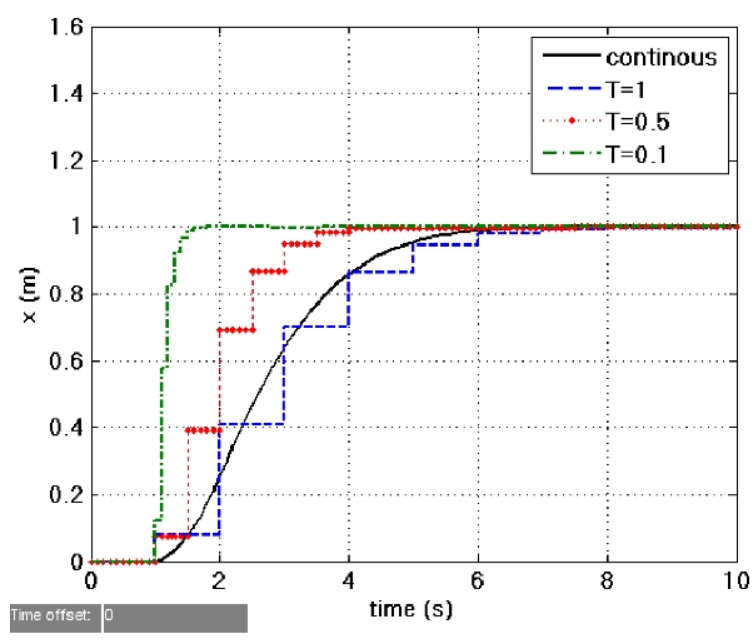

Fig. 2. The displacement of the cart

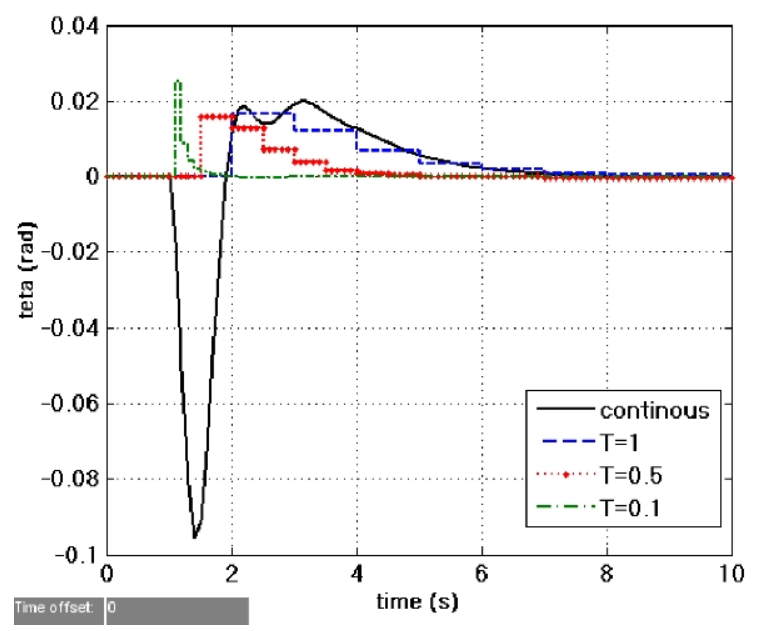

Fig. 3. The angular displacement of the pendulum

As it is seen, the continuous system and the discrete system with sampling time $T=1$, show similar response, while decreasing the sampling time cause the better response of the system.

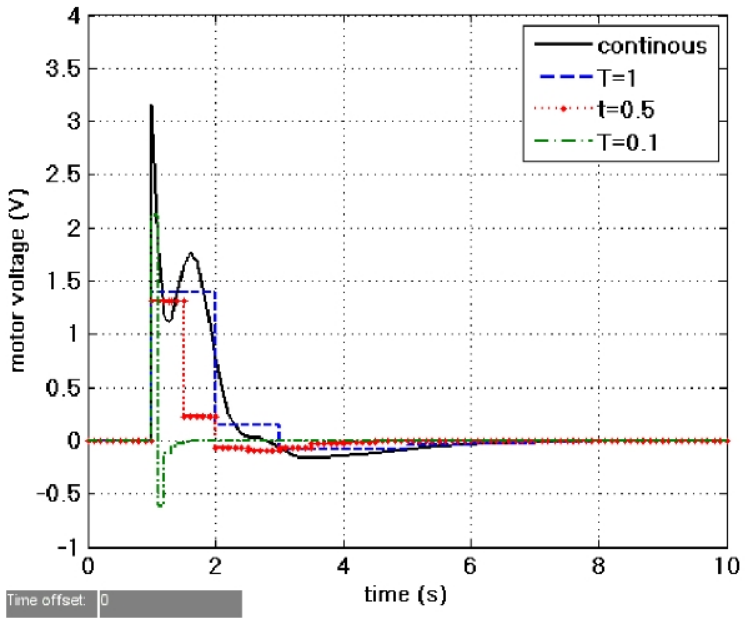

Fig. 4. The Voltage of the motor

\section{CONCLUSION}

This paper has presented a comparison of continuous and discrete optimal control strategy for tracking of a gantry crane system. the nonlinear and linear dynamic of the continuous system have been derived, and the equivalent linear discrete system has been presented. Using, the optimal control formulation for tracking problems, the corresponding optimal feedback laws have been obtained, and the simulation results have been presented. The results have shown that decreasing the sampling time of the discrete model can be resulted in better response.

\section{REFERENCES}

[1] Abdel-Rahman E M, Nayfeh A H, Masoud Z N (2003) Dynamic and Control of Cranes: a Review. $J$ of Vibration and Control, 9: 863-908.

[2] Ahmad M A, Nasir A N K, Raja Ismail R M T, Ramli M S (2010) Control Schemes for Input Tracking and Anti-sway Control of a Gantry Crane. Australian J of Basic and Applied Sciences, 4: 2280-2291.

[3] Ahmad M A (2009) Active Sway Suppression Techniques of a Gantry Crane System. European J of Scientific Research, 27: 322-333.

[4] Dey R, Sinha N, Chaube P, Ghosh S, Ray G (2010) Active Sway Control of a Single Pendulum Gantry Crane System using Output-Delayed Feedback Control Technique. Int. Conf. Control, Automation, Robotics and Vision, 532-536. 
[5] Zrnic N D, Petcovic Z D, Ostric D Z, Brkic A D (1998) On a Method for Defining Horizontal of Gantry Cranes. Proc. of Conf. of Manufac. Engineering, 573-580.

[6] Manning R, Clement J, Kim D, Singhose W (2010) Dynamics and Control of Bridge Cranes Transporting Distributed-Mass Payloads. . J of Dynamics Syestems, Measurement, and Control, 132: 134-142.

[7] Rahn C D, Zhang F, Joshi S, Dawson D M (1999) Asymptotically Stabilizing Angle Feedback for a Flexible Cable Gantry Crane. J of Dynamics Syestems, Measurement, and Control, 121: 563-566.

[8] Hubbell J T, Koch B, McCormick D (1992) Modern Crane Control Enhancements. Seattle, 28-44.

[9] Masoud Z N, Daqaq M F (2006) A Graphical Approach to Input-Shaping Control Design for Container Cranes With Hoist. Trans on Control Systems Technology, 14: 1070-1076.

[10] Ahmad M A, Nasir A N K, Hambali N, Ishak H (2009) Hybrid Input Shaping and PD-type Fuzzy Logic Control Scheme of a Gantry Crane System. Int Conf on Control Applications, Russia, 1051-1056.

[11] Moustafa K A F, Ebeid A M (1988) Nonlinear Modeling and Control of an Overhead Crane Load Sway. ASME Trans on Dynamic Systems, Measurements and Control, 110: 266-271.

[12] Wahyudi M, Jalani J (2005) Design and Implementation of Fuzzy Logic Controller for an
Intelligent Gantry Crane System. Proc of Int Conf on Mechatronics, 345- 351.

[13] Friedland B (1986) Control System Design. McGraw-Hill Book Company.

[14] Ogata K (2002) Discrete-Time Control Systems. Prentice Hall.

[15] Ogata K (2002) Modern Control Engineering. Aeeizb Publisher.

[16] lles S, Koloni F, Matusko J (2011) Linear Matrix Inequalities Based H1 Control of Gantry Crane using Tensor Product Transformation, Int Conf on Process Control, 92- 99.

M. Nazemizadeh was born in Isfahan Iran. He recived his B. Sc. in Mechanical Engineering from the Shahrekord University. He has also obtained his master's degrees in mechanical engineering at the Iran University of Science and Technology. He is now an education instructor in Islamic Azad Universtiy, and he is a $\mathrm{PhD}$ student in mechanical engineering at Amirkabir university of Iran, too.

$\mathrm{He}$ is interested in robotics researches and his works are mainly concentrated on obstacle avoidance and optimal path planning of manipulators and mobile robots. He has published about 15 papers on international and national journals and conferences, until now. 